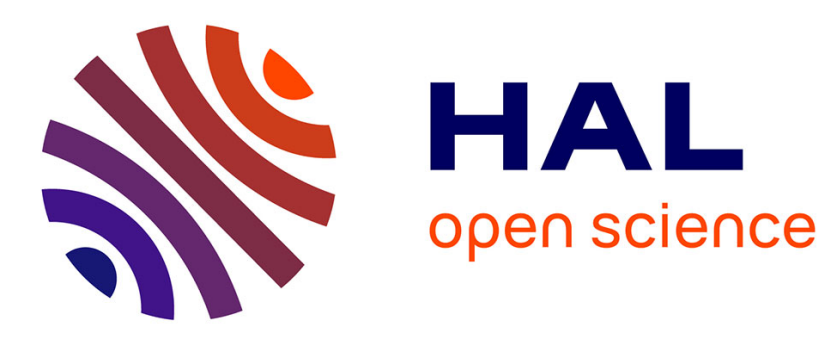

\title{
Flapping-Wing Mechanism for a bird-Sized UAVS: Design, Modeling and Control
}

Christophe Grand, Pascal Martinelli, Jean-Baptiste Mouret, Stéphane Doncieux

\section{To cite this version:}

Christophe Grand, Pascal Martinelli, Jean-Baptiste Mouret, Stéphane Doncieux. Flapping-Wing Mechanism for a bird-Sized UAVS: Design, Modeling and Control. 11th Int. Symposium on ADVANCES IN ROBOT KINEMATICS, 2008, Batz-sur-Mer, France. pp.127-136. hal-01300718

\section{HAL Id: hal-01300718 https://hal.science/hal-01300718}

Submitted on 11 Apr 2016

HAL is a multi-disciplinary open access archive for the deposit and dissemination of scientific research documents, whether they are published or not. The documents may come from teaching and research institutions in France or abroad, or from public or private research centers.
L'archive ouverte pluridisciplinaire HAL, est destinée au dépôt et à la diffusion de documents scientifiques de niveau recherche, publiés ou non, émanant des établissements d'enseignement et de recherche français ou étrangers, des laboratoires publics ou privés. 


\title{
Flapping-wing mechanism for a bird-sized UAVs: design, modeling and control
}

\author{
Ch. Grand ${ }^{1,2}$, P. Martinelli ${ }^{2}$, J.-B. Mouret ${ }^{1}$ and S. Doncieux ${ }^{1}$ \\ ${ }^{1}$ ISIR, Université Pierre et Marie Curie-Paris 6, France \\ ${ }^{2}$ IUT Cachan, Université Paris SUD-Paris 11,France \\ e-mail: grand@isirfr
}

\begin{abstract}
Birds daily execute complex maneuvers out of reach of current UAVs of comparable size. These capabalities are at least partly linked to the efficient flapping kinematics. This article describes the flapping wing mechanism developed within the ROBUR project to create a bird-sized UAV relying on such advanced kinematics.
\end{abstract}

\section{Introduction}

The capabilities of Mini-UAVs have drastically increased thanks to recent advances in terms of energy storage, effector power and electronic miniaturization, but they still remain far below the maneuverability and energy efficiency exhibited by birds and bats. An European kestrel for instance can stay above a point, fly forward at varying speeds, glide or soar to save energy, while demonstrating maneuverability capacities that far exceed those of the most efficient acrobatic aircraft. We have chosen to study flapping-flight for bird-sized UAVs, i.e., with a wing-span ranging from 0.5 to 1 meter and a weight of $500 \mathrm{~g}$.

This article describes current research efforts targeted at designing a flappingwing platform within the ROBUR project ([Doncieux et al., 2006]). Whereas current flapping-flight artifacts show a maneuverability similar to that of fixed-wing engines, our overall objective is to design an aircraft whose capabilities will more closely resemble that of a kestrel, or at least of a pigeon. To this end, the wing kinematics have to be carefully controlled, for instance through the implementation of a neural network-based closed-loop control. As a consequence, the mechanical instantiation of our artificial bird must be able to move the wings on a wide range of periodical and non periodical trajectories, while remaining as energyefficient as possible. This makes the corresponding wing-beat mechanism different from many mechanisms found in the literature ([Pornsin-Sirirak et al., 2001, Vest and Katz, 1999, Raney and Slominski, 2004, Hunt et al., 2005]), because they generate periodical or quasi-sinusoidal movements only. The design of such an improved device is especially challenging since, to properly size its actuators and me- 
chanical parts, we must know the order of magnitude of torques, angle ranges and dimensions, which strongly depend on the wing-beat kinematics and the morphology of the artificial bird. The optimal kinematics, as well as the required degrees of freedom and the required power to fly at a given speed, being still open scientific questions, we want to address them while designing such mechanism.

This article deals with the design, the modeling and the control of the flappingwing mechanism developed within the ROBUR project. In a first part, we briefly describe the results obtained on the optimal design of bird morphology and kinematics. Based on these results the wing beating mechanism that we have designed is then presented. In a second and a third part, the system modeling and its control are described.

\section{Mechanical design}

In a first step, the morphology and kinematics the most adapted to several flying speeds have been sought using an evolutionary algorithm. Figure (1.a) shows the wing panels and their DOFs (dihedral (DI), sweep (SW), shoulder incidence (SINC) and wrist incidence (WINC)). In figure (1.b) possible morphologies corresponding to boundary values of wing area $\left(0.1-0.4 \mathrm{~m}^{2}\right)$ and wing aspect ratio (4.5-10) are illustrated.

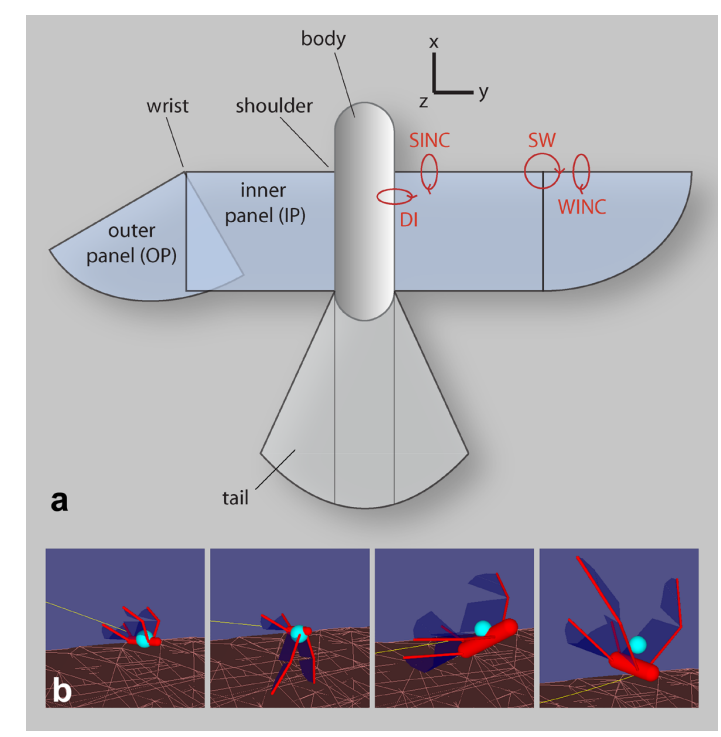

Fig. 1 The morphology of a simulated UAV. 
The results of this optimization give us the first insights of the typical mechanical parameters required for a basic horizontal flight of a $0.5 \mathrm{~kg}$ UAV at different speed ([de Margerie et al., 2007]). Optimization lead to a minimum energy consumption of $20-50 \mathrm{~W} / \mathrm{kg}$ for a $10-12 \mathrm{~m} / \mathrm{s}$ speed, with wing-beat frequencies between 3 to 5 Hz. Table 1 describes the obtained angle ranges for the different DOFs, for Paretooptimal kinematics. This means that the UAV should at least allow the DOFs to move in these ranges for a basic flight along a straight line.

\begin{tabular}{|c|c|c|c|}
\hline Speed $(\mathrm{m} / \mathrm{s})$ & Dihedral & Shoulder incidence & Wrist incidence \\
\hline $6-8$ & $15-50$ & $0-30$ & $10-50$ \\
$10-12$ & $25-45$ & $0-15$ & $8-15$ \\
$16-20$ & $30-65$ & $0-5$ & $1-10$ \\
\hline
\end{tabular}

Table 1 Angular ranges (in degrees) for the three DOFs (wing folding is not represented) for Pareto-optimal individuals.

Capitalizing on these results, we designed a wing-beating mechanism that allows a wide range of dihedral and twist variations, with a high energetic and mechanical efficiency. To reach such capabilities, an innovative mechanism was developed as shown in figure 2 .

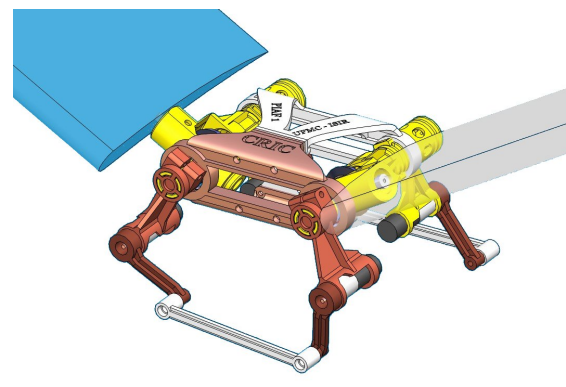

(a) General view

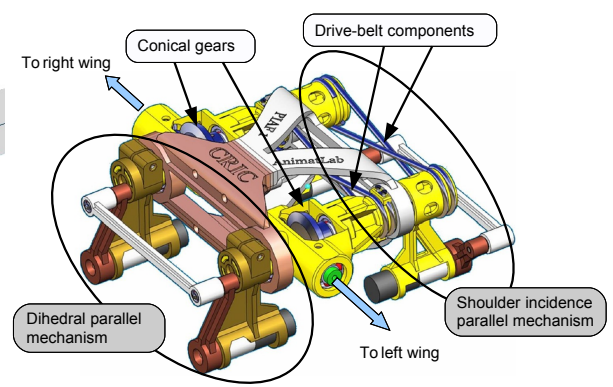

(b) Detailed view with sub-components

Fig. 2 Wing-beating mechanism.

In this mechanism, the wings motion is produced by four position-controlled brushless motors (each motor is about $30 \mathrm{~W}$ power and $100 \mathrm{~g}$ weight). These four motors are associated two by two, constituting a parallel mecanism that uses two connected rod-crank mechanisms. Thus, the wings can be moved to follow an arbitrary trajectory and the power required to execute the quasi-sinusoidal movements is especially low. Each pair of motors is used to control: (1) the dihedral (DI) motion and (2) the shoulder incidence (SINC) motion. 


\section{Kinematical modelling}

The wing-beating system is composed of two identical mechanisms. This elementary part is a parallel system actuated by two motors. The first one, located in the front plane, is directely used to control the dihedral motion of the wings and the second one, located on the rear plane, controls the shoulder incidence motion through two conical gears. Each of these elementary systems is made symmetrical by using a drive-belt component (see figure 2). Thus, in this first prototype, the flapping motion is identical for left and right wings (both for dihedral and twist motion).

The elementary parallel mechanism is composed of five rods connected through six revolute joints. The figure 3 shows the kinematic schema of this parallel mechanism. Joint $J_{1}$ and $J_{2}$ are coupled by the drive-belt, joint $J_{3}$ and $J_{4}$ are actuated by two motors, and the last two joints $\left(J_{5}, J_{6}\right)$ remain free. The mobility index of the mechanism is given by the classical Grubler equation :

$$
m=\sum_{i=1}^{n} f_{i}-3(n-b)
$$

where $b$ is the number of moving bodies, $n$ the number of joints, $f_{i}$ the number of degrees of freedom of each joint $i$. In this case, the mobility index is $m=3$ ( 5 bodies, 6 revolute joints), but the overall system mobility is reduced to $m=2$ when the symmetry condition from the drive-belt is considered. As these two degrees of freedom are actuated by two motors, the mechanism motion is completely constrained.

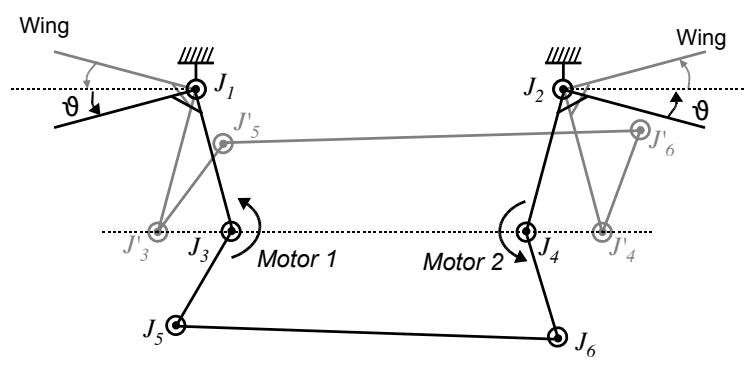

Fig. 3 Kinematic schema of the parallel mechanism

For analysis purpose, the system can be simplified by the kinematically equivalent system depicted in figure 4(b). Because of the symmetrical motion on joint $J_{l}$ and $J_{2}$ (angle $\theta$ ), the motion of points $\mathrm{A}_{3}$ and $\mathrm{A}_{4}$ is constrained to an horizontal axis ( $\mathrm{A}_{i}$ denotes the center of joint $J_{i}$ ). Thus, we can consider only the 3 rods mechanism and express the kinematic relation between $\lambda$ and the input angles $\alpha_{1}$ and $\alpha_{2}$. The solution is obtained by solving the kinematical closure-form equations :

$$
\left\{\begin{array}{l}
b \cos \alpha_{1}+L \cos \gamma-b \cos \alpha_{2}=\lambda \\
b \sin \alpha_{1}+L \sin \gamma-b \sin \alpha_{2}=0
\end{array}\right.
$$


Eliminating $\gamma$ from these equations gives the following expression:

$$
L^{2}=\left(\lambda+b\left(\cos \alpha_{1}-\cos \alpha_{2}\right)\right)^{2}+b^{2}\left(\sin \alpha_{1}-\sin \alpha_{2}\right)^{2}
$$

Then, $\lambda$ can be determined from this second order equation. When the solution existence condition is satisfied $(L>\sqrt{2} b)$, we obtain the following unique solution:

$$
\lambda=\sqrt{L^{2}-b^{2}\left(\sin \alpha_{1}-\sin \alpha_{2}\right)^{2}}+b\left(\cos \alpha_{1}-\cos \alpha_{2}\right)
$$

And considering the schema on the figure 4(a), the wing flapping angle $\theta$ is given as a function of $\lambda$ :

$$
\theta=\sin ^{-1} \frac{L-\lambda}{2 a}
$$

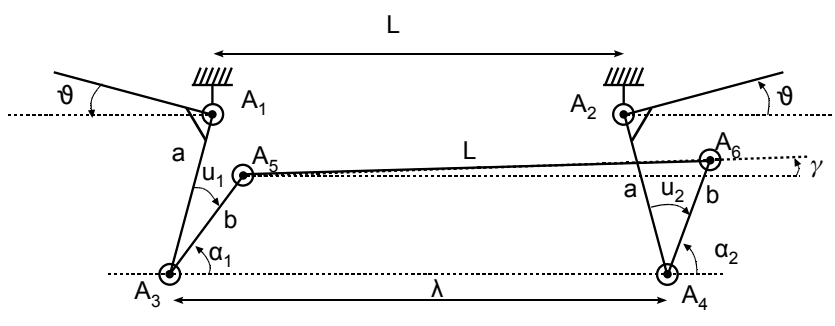

(a) Kinematical parameters

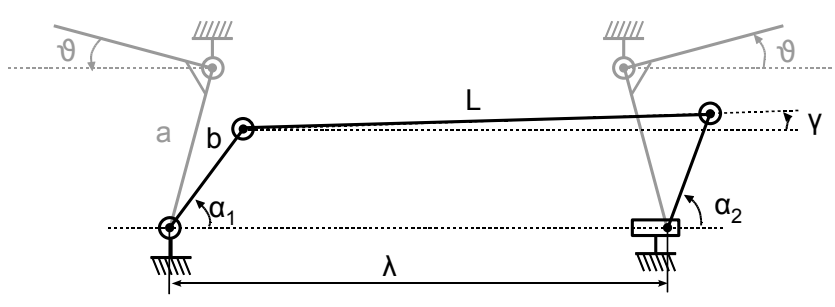

(b) Simplified schema

Fig. 4 Detailed kinematic schema

The reader should notice that the parameters $\alpha_{1}$ and $\alpha_{2}$ are not directly the control inputs. But, if we consider $\mathbf{u}=\left[\begin{array}{ll}u_{1} & u_{2}\end{array}\right]^{\mathrm{t}}$ the input vector composed of the two motor angles (corresponding to the joint angles of $J_{3}$ and $J_{4}$ ), there can be computed as function of the parameters $\alpha_{1}, \alpha_{2}$ and the kinematical configuration of the mechanism characterized by the angle $\theta$ :

$$
\left\{\begin{array}{l}
u_{1}=\left(\alpha_{1}-\frac{\pi}{2}\right)-\theta \\
u_{2}=\left(\alpha_{2}-\frac{\pi}{2}\right)+\theta
\end{array}\right.
$$


However for the mathematical description of the kinematical model, it is more efficient to consider the input parameters $\left(\alpha_{1}, \alpha_{2}\right)$ instead of the motor angles $\left(u_{1}, u_{2}\right)$.

The system presents two control inputs $\alpha_{1}$ and $\alpha_{2}$ for one state parameter $\theta$. So, the mechanism is over-actuated and we need to determine the compatible angles. Thus, let us introduce a new set of input variables $\alpha$ and $\varphi$, respectively the mean input angle and the half-phase angle :

$$
\left\{\begin{array} { l } 
{ \alpha = \frac { 1 } { 2 } ( \alpha _ { 2 } + \alpha _ { 1 } ) } \\
{ \varphi = \frac { 1 } { 2 } ( \alpha _ { 2 } - \alpha _ { 1 } ) }
\end{array} \quad \text { and } \quad \left\{\begin{array}{l}
\alpha_{1}=\alpha-\varphi \\
\alpha_{2}=\alpha+\varphi
\end{array}\right.\right.
$$

The equation (3) becomes:

$$
\lambda=\sqrt{L^{2}-4 b^{2} \cos ^{2} \alpha \sin ^{2} \varphi}+2 b \sin \alpha \sin \varphi
$$

And the relations between the motor angular positions $\left(u_{1}\right.$ and $\left.u_{2}\right)$ and the new variables become:

$$
\left\{\begin{array} { l } 
{ u _ { 1 } = ( \alpha - \frac { \pi } { 2 } ) - ( \theta + \varphi ) } \\
{ u _ { 2 } = ( \alpha - \frac { \pi } { 2 } ) + ( \theta + \varphi ) }
\end{array} \quad \text { and } \quad \left\{\begin{array}{rl}
\alpha & =\frac{1}{2}\left(u_{2}+u_{1}\right)+\frac{\pi}{2} \\
\varphi+\theta & =\frac{1}{2}\left(u_{2}-u_{1}\right)
\end{array}\right.\right.
$$

\section{Motion control of the flapping angle}

In this part, we demonstrate that the previous choice of variables $\alpha$ and $\varphi$ leads to a simplified control model of the wings-flapping motion. It allows to define quasisinusoidal motion given by the desired flapping frequency and amplitude.

The equation (5) can be differentiated with respect to $\alpha$ :

$$
\frac{d \lambda}{d \alpha}=2 b \cos \alpha \sin \varphi\left[1+\frac{2 b \sin \alpha \sin \varphi}{\sqrt{\frac{L^{2}}{4 b^{2}}-\cos ^{2} \alpha \sin ^{2} \varphi}}\right]
$$

By considering the schema depicted in figure 3 , one can conclude that the wing angle $\theta$ is at its extremums when $\lambda$ is also at its extremums. In order to find the extremum positions $\alpha_{\min }$ and $\alpha_{\max }$, the relation $\frac{d \lambda}{d \alpha}=0$ must be solved. This leads to the following solutions:

$$
\left\{\begin{array}{l}
\cos \alpha=0 \\
\sin \varphi= \pm \frac{L}{2 b}
\end{array}\right.
$$

Thus, for any values of $\varphi$ there are only two extremum positions at each periode that are solutions of $\cos \alpha=0$. When introducing this result in the equations (5) and (4), we can find the maximum values for $\lambda$ and consequently for $\theta$ :

$$
\left\{\begin{array}{l}
\lambda_{\max }=L \pm 2 b \sin \varphi \\
a \sin \theta_{\max }=b \sin \varphi
\end{array}\right.
$$


The figure 5 shows some trajectories $\theta$ obtained for different phase angles $\varphi$. The motion is quasi-sinusoidal, its amplitude depends on $\varphi$ and its frequency can be modulated thanks to the velocity term $\dot{\alpha}$. So, if the desired quasi-sinusoidal trajectory is specified by its frequency $f_{\theta}$ and its amplitude $\theta_{\max }$, the velocity $\dot{\alpha}$ and the phase are determined as follows:

$$
\left\{\begin{array}{l}
\dot{\alpha}=2 \pi f_{\theta} \\
\varphi=\sin ^{-1}\left(\frac{a}{b} \sin \theta_{\text {max }}\right)
\end{array}\right.
$$

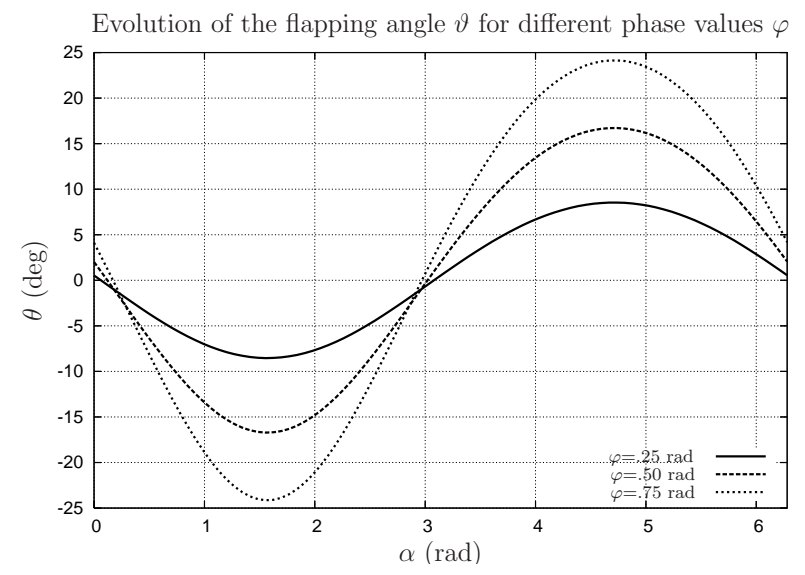

Fig. 5 Exemple of $\theta$ trajectory obtained with kinematic based control

In order to extent this simple quasi-sinusoidal control to an arbitrary flapping motion, we need to investigate the differential kinematic model. The flapping velocity is given by $\dot{\theta}$ that can be expressed as a fonction of the input velocities $(\dot{\alpha}, \dot{\varphi})$ :

$$
\dot{\theta}=\frac{d \theta}{d \lambda}\left(\frac{d \lambda}{d \alpha} \dot{\alpha}+\frac{d \lambda}{d \varphi} \dot{\varphi}\right)=J_{\alpha} \dot{\alpha}+J_{\varphi} \dot{\varphi}
$$

The corresponding jacobian terms are determined from equations (4) and (6):

$$
\left\{\begin{array}{l}
J_{\alpha}=\frac{d \theta}{d \lambda} \frac{d \lambda}{d \alpha}=-\frac{b \cos \alpha \sin \varphi}{a \sqrt{1-\left(\frac{L-\lambda}{2 a}\right)^{2}}}\left[1+\frac{2 b \sin \alpha \sin \varphi}{\sqrt{\frac{L^{2}}{4 b^{2}}-\cos ^{2} \alpha \sin ^{2} \varphi}}\right] \\
J_{\varphi}=\frac{d \theta}{d \lambda} \frac{d \lambda}{d \varphi}=-\frac{b \sin \alpha \cos \varphi}{a \sqrt{1-\left(\frac{L-\lambda}{2 a}\right)^{2}}}\left[1-\frac{2 b \cos \alpha \cot \alpha \sin \varphi}{\sqrt{\frac{L^{2}}{4 b^{2}}-\cos ^{2} \alpha \sin ^{2} \varphi}}\right]
\end{array}\right.
$$

Then, the control principle is to compute the instantanous velocities terms $(\dot{\alpha}, \dot{\varphi})$ as a fonction of the desired flapping velocity $\dot{\theta}^{c}$. This desired velocity becomes the 
control input used to follow a desired trajectory $\theta(t)$ with a classical feedforward controller:

$$
\dot{\theta}^{c}=K_{1}\left(\theta-\theta^{m}\right)+\dot{\theta}
$$

where $\theta^{m}$ is the mesured flapping angle, $K_{1}$ is a positive gain and $\dot{\theta}$ is the feedforward velocity computed from the trajectory $\theta(t)$. Then, the control law becomes:

$$
\left\{\begin{array}{l}
\dot{\varphi}=K_{2}\left(\varphi^{d}-\varphi^{m}\right) \\
\dot{\alpha}=\frac{1}{J_{\alpha}}\left(\dot{\theta}^{c}-J_{\varphi} \dot{\varphi}\right)
\end{array}\right.
$$

where $\varphi^{d}$ is the phase angle determined from the maximum absolute values of the flapping angle trajectory $\theta(t)$ on a given time horizon.

\section{Conclusion}

These results constitute the first stones of a long work towards a fully maneuverable flapping-wing UAV. Kinematical and morphological data from an evolutionary optimization process have been used to properly dimension a flapping-mechanism able to move the wing dihedral and incidence to follow arbitrary kinematics. The kinematic model of this innovative parallel mechanism has been detailed in this paper. Based on this model, a simple control law for quasi-sinusoidal motions has been developed. Its extension to a velocity model based controller that is able to follow various cyclic trajectories has been proposed.

\section{References}

[Doncieux et al., 2006] Doncieux, S et al. (2006), Building an Artificial Bird: Goals and Accomplishments of the ROBUR Project, European Micro Aerial Vehicles (EMAV).

[Pornsin-Sirirak et al., 2001] Pornsin-Sirirak, T.N. and Tai, Y.C. and Ho, C.M. and Keennon, M. (2001), Microbat: A Palm-Sized Electrically Powered Ornithopter, Proceedings of NASA/JPL Workshop on Biomorphic Robotics.

[Vest and Katz, 1999] Vest, M.S. and Katz J. (1999), Aerodynamic Study of a Flapping-Wing Micro-UAV, 37th AIAA Aerospace Sciences Meeting and Exhibit.

[Raney and Slominski, 2004] Raney, D.L. and Slominski, E.C. (2004), Mechanization and Control Concepts for Biologically Inspired Micro Air Vehicles, Journal of Aircraft, Vol 41:6, pp 1257-1265

[Hunt et al., 2005] Hunt, R. and Hornby, G.S. and Lohn, J.D. (2004), Toward evolved flight, Proceedings of the 2005 conference on Genetic and evolutionary computation, pp 957-964.

[de Margerie et al., 2007] de Margerie, E., Mouret, J.-B., Doncieux, S., and Meyer, J.-A. (2007). Artificial evolution of the morphology and kinematics in a flapping-wing mini UAV. Bioinspir. Biomim., Vol 2, 65-82. 